\title{
ENHANCING PARAMETRIC SENSITIVITY USING MODE LOCALIZATION IN ELECTRICALLY COUPLED MEMS RESONATORS
}

\author{
P. Thiruvenkatanathan, J. Yan, J.E-Y. Lee, and A.A. Seshia \\ Department of Engineering, Trumpington Street, University of Cambridge, Cambridge, UK
}

\begin{abstract}
We use vibration localization as a sensitive means of detecting small perturbations in stiffness in a pair of weakly coupled micromechanical resonators. For the first time, the variation in the eigenstates is studied by electrostatically coupling nearly identical resonators to allow for stronger localization of vibrational energy due to perturbations in stiffness. Eigenstate variations that are orders of magnitude greater than corresponding shifts in resonant frequency for an induced stiffness perturbation are experimentally demonstrated. Such high, voltagetunable parametric sensitivities together with the added advantage of intrinsic common mode rejection pave the way to a new paradigm of mechanical sensing.
\end{abstract}

\section{KEYWORDS}

Mode localization, resonator, electrical coupling, parametric sensitivity.

\section{INTRODUCTION}

Over the past decade, resonant frequency based detection has emerged as a promising technique for detecting small, linear parametric variations in the structural properties of micromechanical sensors [1]-[3]. In these sensors the output corresponds to a shift in the resonant frequency of a vibrating micromechanical structure when subjected to small perturbations in either its stiffness or mass. The naturally high frequency sensitivity [3] and the quasi-digital nature of the signal [1], makes this method of detection particularly attractive for sensing applications. In what follows, we propose the use of an alternate method of detection wherein the dramatic shifts in the eigenstates caused by vibration localization in an array of weakly coupled resonators are used as a means of enhancing the parametric sensitivity even further than that attained using the resonant frequency shift approach.

In an array of identical resonators coupled through weak springs, a small perturbation in the structural properties of one of the resonators can strongly impact coupled oscillations resulting in (sometimes severe) confinement of vibration energy to small geometric regions of the system [4], [5]. The extent of this vibration energy confinement or localization depends not only on the magnitude of the periodicity breaking irregularity but also on the strength of internal coupling between the resonators, with weaker coupling leading to stronger localizations. This phenomenon consequently results in drastic variations in the eigenstates that may be as high as orders of magnitude greater than corresponding shifts in resonant frequency induced by the same structural perturbation.

Spletzer et al. recently proposed the application of this principle for sensing small variations in mass for an array of nearly identical microcantilevers that are weakly coupled using small mechanical overhangs near the base of the cantilevers [6], [7]. In this paper, the phenomenon of mode localization is employed as a highly sensitive means of detecting small variations in stiffness. Furthermore, the vibration localization is studied by electrically coupling the resonators, allowing for significantly weaker coupling spring constants and the possibility for stronger, tunable localization of the vibration modes. Nearly three orders of magnitude improvement in signal sensitivity over resonant frequency variations are experimentally demonstrated. A close match between theoretical predictions and experimental observations is also shown, validating the potential of mode localization for use in a sensing capacity.

\section{THEORY}

In order to understand the underlying physics, consider two identical resonators coupled through a weak spring $\left(k_{c}\right)$ as represented in the discretized model shown in Fig. 1 .

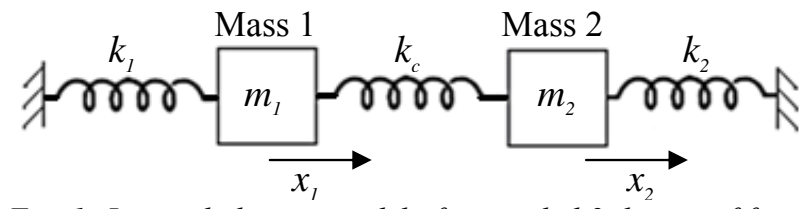

Fig. 1: Lumped element model of a coupled 2 degree of freedom spring-mass system.

When the two coupled structures are identical (i.e., when $m_{1}=m_{2}=m ; k_{1}=k_{2}=k$ ), the system is symmetric about the coupling spring $\left(k_{c}\right)$ and the free vibration response of the undamped system is given by

$$
\begin{aligned}
& m \ddot{x}_{1}+k x_{1}+k_{c}\left(x_{1}-x_{2}\right)=0 \\
& m \ddot{x}_{2}+k x_{2}+k_{c}\left(x_{2}-x_{1}\right)=0
\end{aligned}
$$

Taking $\left[\begin{array}{ll}x_{1} & x_{2}\end{array}\right]^{T}=u_{0 n} e^{i \omega t}$ (for $n=1,2$ ), the associated non-dimensionalized eigenvalue problem can be expressed as

$$
K_{0} u_{0 n}=\lambda_{0 n} M_{0} u_{0 n}(\text { for } n=1,2)
$$


for the mode shape $u_{0 n}$, where $\lambda_{0 n}=\frac{\omega_{n}^{2}}{\mathrm{k} / \mathrm{m}}$ and $K_{0}$ and $M_{0}$ represent the stiffness and mass matrices, given by

$$
K_{0}=\left[\begin{array}{cc}
1+\frac{k_{c}}{k} & -\frac{k_{c}}{k} \\
-\frac{k_{c}}{k} & 1+\frac{k_{c}}{k}
\end{array}\right] ; M_{0}=\left[\begin{array}{ll}
1 & 0 \\
0 & 1
\end{array}\right]
$$

The initial vibration response of this unperturbed, perfectly symmetric system, may then be obtained by solving equation (2) to get

$$
\begin{gathered}
\lambda_{01}=1, u_{01}=\frac{1}{\sqrt{2}}\left[\begin{array}{l}
1 \\
1
\end{array}\right] \\
\lambda_{02}=1+2 \frac{k_{c}}{k}, u_{02}=\frac{1}{\sqrt{2}}\left[\begin{array}{c}
1 \\
-1
\end{array}\right]
\end{gathered}
$$

A slight perturbation in stiffness or mass on either of the coupled structures results in a break in the symmetry, thereby causing a modification in both the eigenvalues and eigenvectors. The new modes and eigenvalues of the slightly perturbed system may then be obtained using the perturbation approach [8] by solving

$$
K u_{n}=\lambda_{n} M u_{n}(\text { for } n=1,2)
$$

where the symmetric perturbed stiffness and mass matrices may be expressed as

$$
K=K_{0}+\delta K+\ldots ; M=M_{0}+\delta M+\ldots
$$

and the perturbed eigenvalues and eigenvectors may be represented by

$$
\lambda_{n}=\lambda_{0 n}+\delta \lambda_{n}+\ldots ; u_{n}=u_{0 n}+\delta u_{n}+\ldots
$$

Substituting equations (6), and (7) into equation (5) for the case when there is a small perturbation in the stiffness of one of the structures $\left(k_{2 \text { new }}=k_{2}+\Delta k\right.$ where $\Delta k$ denotes the change in stiffness) and no perturbation in mass yields:

$$
\frac{\left|u_{n}-u_{0 n}\right|}{\left|u_{0 n}\right|} \approx\left|\frac{\Delta k}{4 k_{c}}\right| ;(n=1,2) \text {. }
$$

Comparing this with the approach of measuring relative shift in the resonant frequency (refer equation (9)), it can be observed that for any value of $k c<k / 2$, the relative shift in the mode shape is greater than that of the resonant frequency:

$$
\frac{\left|f-f_{0}\right|}{\left|f_{0}\right|} \approx\left|\frac{\Delta k}{2 k}\right|
$$

Hence, by weakening the strength of the coupling spring between the two structures, relative shifts in the eigenstates can be orders of magnitude greater than resonant frequency variations. Such drastic shifts in the eigenstates are used here as a sensitive means of detecting very small perturbations in stiffness in a pair of nearly identical, electrically coupled resonant structures.
Electrostatic coupling is employed as this allows for significantly weaker coupling spring constants $\left(k_{c}\right)$ than those that can be attained mechanically and in consequence, the possibility of greater variations in the vibration modes for a given stiffness perturbation.

When two nearly identical resonators separated by an electrical coupling gap are subjected to different DC polarization voltages, the displacement-dependent component of the attractive electrostatic force generated between the resonators results in the formation of a negative electrostatic spring, the magnitude of which is given by [9]

$$
k_{c}=-\frac{\partial F}{\partial x}=-\frac{\varepsilon_{0} A}{g^{3}}\left(\Delta V^{2}\right)
$$

where $\varepsilon_{0}$ represents the permittivity of free space; $A$, the cross section area of the coupling gap; $g$, the coupling gap and $\Delta V$, the difference in DC polarization voltages of the two resonators.

\section{IMPLEMENTATION AND VERIFICATION}

The concept is demonstrated using a pair of electrically coupled, nearly identical double ended tuning forks (DETF). The device was fabricated in a commercial SOI-MEMS foundry process through MEMSCAP Inc., USA. Each of the tines was designed to be $25 \mu \mathrm{m}$ thick, $300 \mu \mathrm{m}$ long and $6 \mu \mathrm{m}$ wide with a gap of $6 \mu \mathrm{m}$ between the tines. All features including the drive and coupling gaps were designed to be $2 \mu \mathrm{m}$ wide in both resonator configurations.

Each of the resonators was driven and sensed using capacitive transduction. The actuation was achieved using parallel plates of equal dimensions $(260 \mu \mathrm{m}$ long, $6 \mu \mathrm{m}$ wide, $25 \mu \mathrm{m}$ thick), attached to either side as shown in the optical micrograph (Fig. 2).

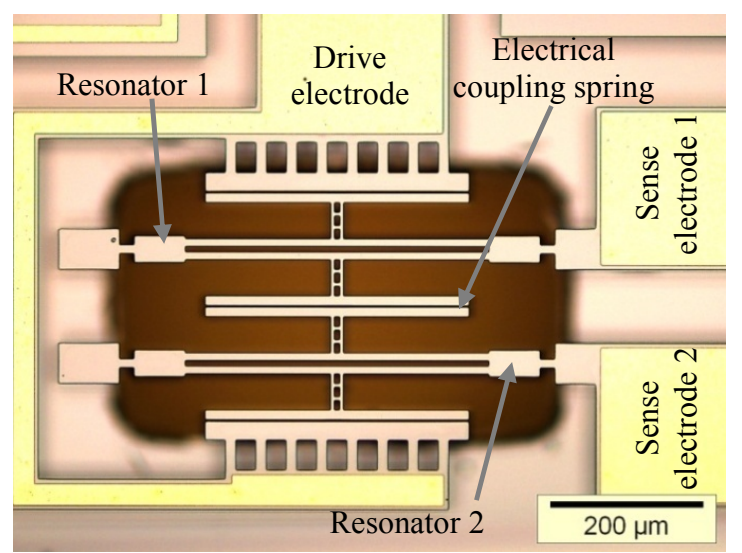

Fig. 2 Optical micrograph of electrically coupled DETF resonators

The resonators were actuated in the out-of-phase mode of vibration. The fabricated devices were tested under 
vacuum $(\approx 50 m$ Torr $)$ in a custom vacuum chamber. A schematic of the measurement setup is shown in Fig. 3.

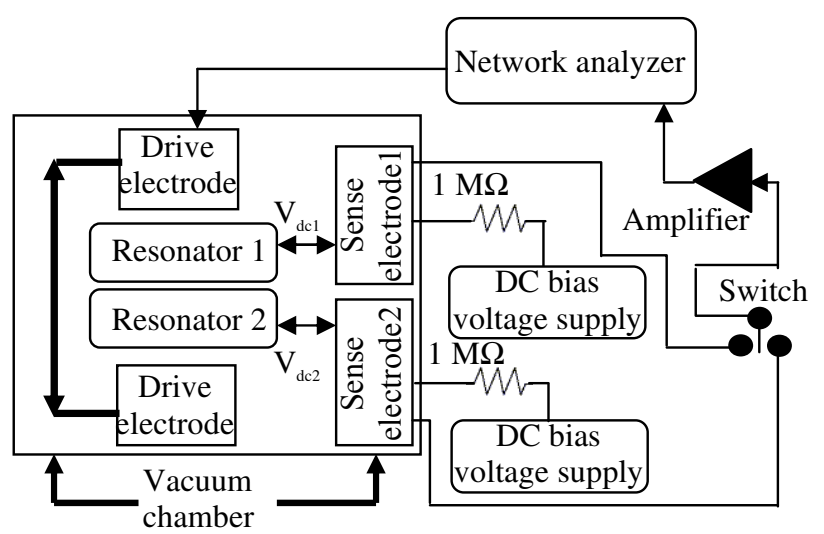

Fig. 3: Optical micrograph of electrically coupled DETF resonators

DC polarization voltages corresponding to $-5 \mathrm{~V}$ and $+5 \mathrm{~V}$ were initially applied on resonators 1 and 2 respectively of the DETF configuration (refer Fig. 3). This should create the unperturbed symmetric condition of the initial eigenvalue problem represented in equation (3). The voltage on resonator 2 was then lowered in steps of one volt, thereby altering both the coupling spring stiffness and the effective stiffness of resonator 2 relative to that of resonator 1 due to the electrostatic spring softening effect. The variation in the eigenstates of the system owing to induced symmetry breaking perturbations in the stiffness of resonator 2 was then deduced from the Y parameter frequency responses of each of the two coupled resonators extracted from the S21 parameter measured on a network analyzer. The DC bias voltage normalized transmission responses of resonators 1 and 2 of the DETF configuration for the different bias conditions are shown in Figs. 4 (a) and (b) respectively.

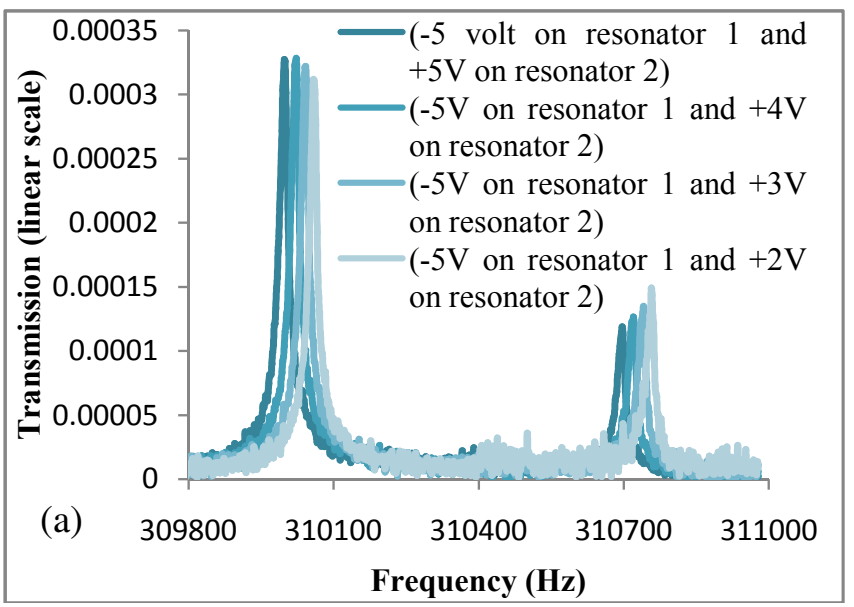

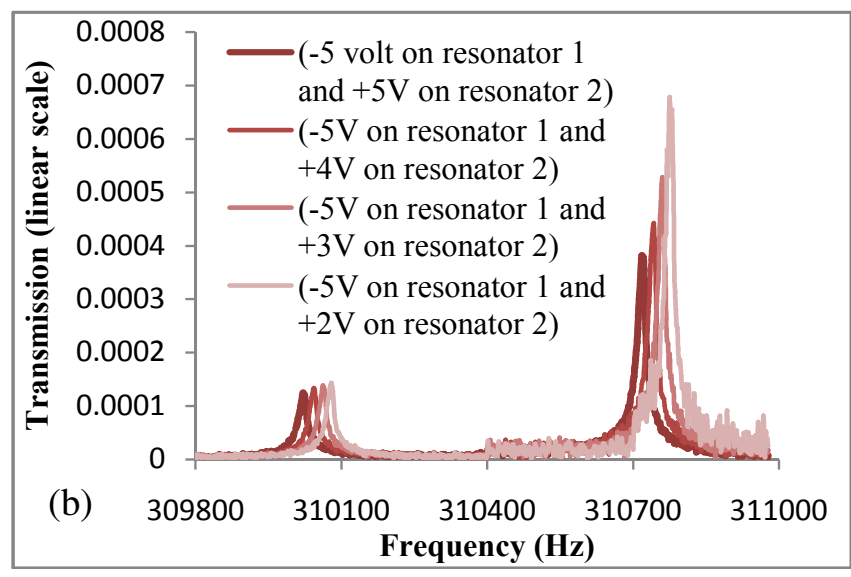

Fig. 4: Experimentally observed (DC voltage normalized) frequency response of resonator 1 (a) and resonator 2 (b).

From Figs. 4 (a) and (b), the relative shift in the eigenstates can be deduced as elaborated in table 1 .

It can be noticed that the variations in the eigenstates are two orders of magnitude greater than corresponding shifts in the resonant frequency for the same parametric perturbation in stiffness. Furthermore, altering the DC polarization voltages allows for varying the strength of the electrostatic coupling spring between the two resonators, thereby enabling the manipulation of the parametric sensitivity for a given structural perturbation as seen from Fig. 4 and table 1 .

To achieve high parametric sensitivities, the two tuning fork resonators must be identical. However, fabrication tolerances limit perfect matching of the resonators, subsequently resulting in slight deviations between the observed variations and theoretical predictions. This can be observed from Fig. 5 which compares the experimentally measured variation in the eigenstates of the system with theoretical predictions. The localization of the vibration modes owing to initial mechanical asymmetries can be observed from the eigenstates measured at the unperturbed condition corresponding to the application of $-5 \mathrm{~V}$ on resonator 1 and $+5 \mathrm{~V}$ on resonator 2 .

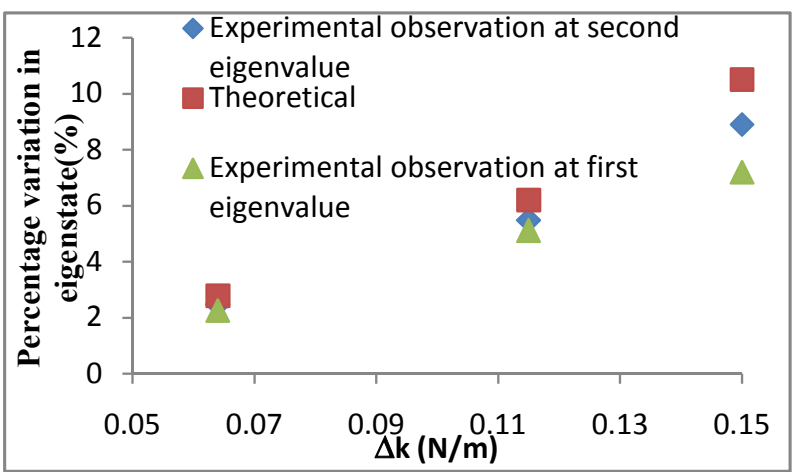

Fig. 5: Comparison between experimentally observed variation in mode shape and theoretical estimates. 


\section{ADVANTAGES OF MODE LOCALIZED SENSING}

Measuring the shifts in the eigenstates in electrically coupled micromechanical resonators offers several advantages over the method of measuring resonant frequency variations. Firstly, orders of magnitude enhancement in parametric sensitivity is obtained. However, it is to be noted here that some of this advantage might be lost as the minimum amplitude shift that can be resolved may be lower than that of frequency. This is a subject of continuing research. Secondly, the realization of a voltage controllable coupling spring allows for tuning the parametric sensitivity as well as compensating for initial fabrication tolerances. Thirdly, such sensors can offer the important advantage of intrinsic common mode rejection. This stems from the fact that the output of the device corresponds to the eigenstate of the system, which is deduced from the amplitudes of both the coupled resonators at the eigenvalues. Any environmental drift should therefore affect both the identical resonators simultaneously to the same degree, thereby allowing for the cancellation of these effects.

\section{CONCLUSIONS}

This paper proposes vibration mode localization as a highly sensitive method of detecting small parametric variations in the structural properties of electrically coupled micromechanical resonators. Variations in eigenstates that are nearly three orders of magnitude greater than corresponding shifts in the resonant frequency are experimentally demonstrated using this approach. It is envisaged that parametric sensitivities as high as four orders of magnitude greater than resonant frequency variations can be obtained by using this method of sensing. Such high, tunable sensitivities together with the common mode rejection capabilities of this sensing paradigm make it an attractive alternative to the more conventional resonant frequency shift sensor approach.

\section{REFERENCES}

[1] A. A. Seshia, M. Palaniapan, T. A. Roessig, R. T. Howe, R. W. Gooch, T. R. Schimert, and S. Montague, "A Vacuum Packaged Surface Micromachined Resonant Accelerometer," $J$. Microelectromech. Syst., vol. 11, pp. 784 -793, 2002.

[2] M. Esashi, "Resonant sensors by silicon micromachining," in Proc. 1996 IEEE Int. Freq. Control Symp., 1996, pp. 609-614.

[3] J. E.-Y. Lee, B. Bahreyni, Y. Zhu, and A. A. Seshia, "Ultrasensitive mass balance based on a bulk acoustic mode single-crystal silicon resonator," Appl. Phys. Lett., vol. 91, pp. 234103, 2007.

[4] C. H. Hodges, "Confinement of vibration by structural irregularity," J. Sound Vib., vol. 82, pp. 441-424,1982.

[5] C. Pierre, "Mode localization and eigenvalue loci veering phenomena in disordered structures," J. Sound Vib., vol. 126, pp. 485-502, 1988.

[6] M. Spletzer, A. Raman, H. Sumali, and J. P. Sullivan, "Highly sensitive mass detection and identification using vibration localization in coupled microcantilever arrays," Appl. Phys. Lett., vol. 92, pp. 114102, 2008.

[7] M. Spletzer, A. Raman, Al. Q. Wu, X. Xu, and R. Reifenberger, "Ultrasensitive mass sensing using mode localization in coupled microcantilevers," Appl. Phys. Lett., vol. 88, pp. 254102, 2006.

[8] R. Courant, and D. Hilbert, Methods of Mathematical Physics, vol.1, Interscience, New York, 1953.

[9] S. Pourkamali, and F. Ayazi, "Electrically coupled MEMS bandpass filters Part II. Without coupling element," Sens. Actuators, Phys. A, vol. 122, pp. 317$325,2005$.

\section{CONTACT}

*Dr. A.A.Seshia, tel: +44-1223-332755; aas41@cam.ac.uk

\begin{tabular}{|c|c|c|c|c|c|c|c|c|}
\hline & \multicolumn{2}{|c|}{$\begin{array}{l}-5 V \text { on resonatorl and } \\
+5 V \text { on resonator } 2\end{array}$} & \multicolumn{2}{|c|}{$\begin{array}{l}-5 V \text { on resonator } 1 \text { and } \\
+4 V \text { on resonator } 2\end{array}$} & \multicolumn{2}{|c|}{$\begin{array}{l}-5 V \text { on resonatorl and } \\
+3 V \text { on resonator } 2\end{array}$} & \multicolumn{2}{|c|}{$\begin{array}{l}-5 V \text { on resonatorl and } \\
+2 V \text { on resonator } 2\end{array}$} \\
\hline & Mode 1 & Mode 2 & Mode 1 & Mode 2 & Mode 1 & Mode 2 & Mode 1 & Mode 2 \\
\hline Eigenstate & {$\left[\begin{array}{c}0.00033 \\
-0.000125\end{array}\right]$} & {$\left[\begin{array}{l}0.00012 \\
0.00038\end{array}\right]$} & {$\left[\begin{array}{c}0.00033 \\
0.000132\end{array}\right]$} & {$\left[\begin{array}{c}0.000127 \\
0.00044\end{array}\right]$} & {$\left[\begin{array}{c}0.00033 \\
0.000139\end{array}\right]$} & {$\left[\begin{array}{l}0.000135 \\
0.000527\end{array}\right]$} & {$\left[\begin{array}{c}0.00033 \\
0.000142\end{array}\right]$} & {$\left[\begin{array}{l}0.00015 \\
0.00068\end{array}\right]$} \\
\hline $\begin{array}{l}\text { Normalized } \\
\text { eigenstate }\end{array}$ & {$\left[\begin{array}{c}0.935 \\
0.35\end{array}\right]$} & {$\left[\begin{array}{l}0.301 \\
0.954\end{array}\right]$} & {$\left[\begin{array}{l}0.928 \\
0.371\end{array}\right]$} & {$\left[\begin{array}{c}0.277 \\
0.96\end{array}\right]$} & {$\left[\begin{array}{l}0.917 \\
0.398\end{array}\right]$} & {$\left[\begin{array}{l}0.248 \\
0.968\end{array}\right]$} & {$\left[\begin{array}{l}0.909 \\
0.416\end{array}\right]$} & {$\left[\begin{array}{l}0.215 \\
0.977\end{array}\right]$} \\
\hline $\begin{array}{l}\text { Relative shift in } \\
\text { eigenstate }\end{array}$ & - & - & {$\left[\begin{array}{l}0.007 \\
0.021\end{array}\right]$} & $\begin{array}{l}0.024 \\
0.006\end{array}$ & $\left.\begin{array}{l}0.018 \\
0.048\end{array}\right]$ & {$\left[\begin{array}{l}0.053 \\
0.014\end{array}\right]$} & {$\left[\begin{array}{l}0.026 \\
0.066\end{array}\right]$} & $\left.\begin{array}{l}0.086 \\
0.023\end{array}\right]$ \\
\hline $\begin{array}{c}\text { Variation in } \\
\text { eigenstate (\%) }\end{array}$ & - & - & $2.21 \%$ & $2.47 \%$ & $5.1 \%$ & $5.48 \%$ & $7.2 \%$ & $8.9 \%$ \\
\hline $\begin{array}{c}\text { Resonant frequency } \\
\text { shift }(\%)\end{array}$ & - & - & $0.00735 \%$ & $0.00732 \%$ & $0.013 \%$ & $0.014 \%$ & $0.019 \%$ & $0.019 \%$ \\
\hline
\end{tabular}

Table 1: Variation of the eigenstates of resonator 1 and 2 for variations in the DC bias on resonator 2 